\title{
APPROXIMATION BY MEDIANTS
}

\author{
WIEB BOSMA
}

\begin{abstract}
The distribution is determined of some sequences that measure how well a number is approximated by its mediants (or intermediate continued fraction convergents). The connection with a theorem of Fatou, as well as a new proof of this, is given.
\end{abstract}

\section{INTRODUCTION}

Let $x$ denote an irrational number. From the expansion of $x$ into a regular continued fraction

$$
x=B_{0}+\frac{1}{B_{1}+\frac{1}{B_{2}+\cdots}}=\left[B_{0} ; B_{1}, B_{2}, \ldots\right]
$$

one gets the convergents $P_{n} / Q_{n}$ of $x$ by truncation,

$$
\frac{P_{n}}{Q_{n}}=\left[B_{0} ; B_{1}, B_{2}, \ldots, B_{n}\right], \quad n \geq 0 .
$$

These convergents satisfy the relation

$$
\frac{P_{n}}{Q_{n}}=\frac{B_{n} P_{n-1}+P_{n-2}}{B_{n} Q_{n-1}+Q_{n-2}}, \quad n \geq 2,
$$

and provide very good approximations to $x$; for instance, defining $\left\{\Theta_{n}(x)\right\}_{n=0}^{\infty}$ by

$$
\left|x-\frac{P_{n}}{Q_{n}}\right|=\frac{\Theta_{n}(x)}{Q_{n}^{2}},
$$

it is a classical result that $\Theta_{n}(x) \leq 1$ always holds. In [1] it was shown that for almost all $x$ the sequence $\left\{\Theta_{n}(x)\right\}_{n=0}^{\infty}$ has a limiting distribution $\frac{1}{\log 2} F(z)$, where

$$
F(z)= \begin{cases}0, & \text { for } z \leq 0 \\ z, & \text { for } 0 \leq z \leq \frac{1}{2} \\ 1-z+\log (2 z), & \text { for } \frac{1}{2} \leq z \leq 1 \\ \log 2, & \text { for } 1 \leq z\end{cases}
$$

Received May 20, 1988.

1980 Mathematics Subject Classification (1985 Revision). Primary 11J70, 11K50.

Key words and phrases. Continued fractions, Diophantine approximation. 
Here we will consider a similar question for the mediants (or secondary convergents, or intermediate convergents) of $x$; these are defined by

$$
\frac{L_{n}^{(B)}}{M_{n}^{(B)}}=\frac{B P_{n-1}+P_{n-2}}{B Q_{n-1}+Q_{n-2}}
$$

for integers $B, 0<B<B_{n} \quad(n \geq 2)$. In particular, we will derive in $\S 1$ for almost all $x$ the limiting distribution of the sequences $\left\{\Theta_{n}^{(B)}(x)\right\}_{n=0}^{\infty}$ for every $B$, where $\Theta_{n}^{(B)}(x)$ is given by

$$
\left|x-\frac{L_{n}^{(B)}}{M_{n}^{(B)}}\right|=\frac{\Theta_{n}^{(B)}(x)}{\left(M_{n}^{(B)}\right)^{2}} .
$$

Note that some care is needed because $L_{n}^{(B)} / M_{n}^{(B)}$ and hence $\Theta_{n}^{(B)}$ does not exist for every $n$ and $B$. The values of $\Theta_{n}^{(B)}$ are not bounded by 1 but satisfy

$$
\frac{B}{B+1} \leq \Theta_{n}^{(B)} \leq B+1 ;
$$

thus these values are uniformly bounded for fixed $x$ if and only if the partial quotients $B_{n}$ are bounded. In $\S 1$ we study the distribution of $\Theta_{n}^{(B)}$ for fixed $B$. In order to be able to study the distribution of the values of $\Theta_{n}^{(B)}$ for all $B$ simultaneously (in $\S 2$ ), we will consider sets of the form $\{\Theta \mid \Theta \leq C\}$ (for any positive real constant $C$ ), with $\Theta=Q|Q x-P|$, where $P / Q$ ranges over the rationals that are either convergents or mediants of $x$. Finally, in $\S 3$ and $\S 4$ we collect some (previously known) results, especially concerning the approximation by nearest mediants, that follow from the method employed. In particular, we show how to retrieve Fatou's theorem, stating that every rational number $P / Q$ for which $Q|Q x-P| \leq 1$ is either a convergent or a nearest mediant of $x$.

In the following we will always assume rationals $P / Q$ (and $L / M$ ) to be in lowest terms, i.e., that $\operatorname{gcd}(P, Q)=1$ and that $Q>0$. Whenever a result is stated for almost all $x$, this is meant to be in the Lebesgue sense.

\section{APPROXIMATION BY MEDIANTS}

The main tool we will use is a variation on a theme that first appeared in [1] and was used in several papers thereafter. The theme consists of considering the sequence $\left\{\left(T_{n}(x), V_{n}(x)\right)\right\}_{n=0}^{\infty}$ for an irrational number $x$, where $T_{n}(x)$ is given by

$$
T_{n}=T_{n}(x)=\left[0 ; B_{n+1}, B_{n+2}, \ldots\right]
$$

and $V_{n}(x)$ by

$$
V_{n}=V_{n}(x)=\left[0 ; B_{n}, B_{n-1}, \ldots, B_{1}\right],
$$

with $B_{i}$ as in (0.1). For every $x$ and every $n$, the pair $\left(T_{n}(x), V_{n}(x)\right) \in[0,1] \times$ $[0,1]$, and for almost all $x$ the sequence $\left\{\left(T_{n}(x), V_{n}(x)\right)\right\}_{n=0}^{\infty}$ is distributed over the unit square with density function

$$
\frac{1}{\log 2} \frac{1}{(1+T V)^{2}} \text {. }
$$


Basically, this is a consequence of the fact that

$$
(\mathscr{M}, \mathscr{B}, \mu, \mathscr{T}) \text { forms an ergodic system; }
$$

here $\mathscr{M}$ is the unit square and $\mathscr{T}$ acts on $\mathscr{M}$ by

$$
\mathscr{T}(x, y)=\left(\frac{1}{x}-\left\lfloor\frac{1}{x}\right\rfloor, \frac{1}{\left\lfloor\frac{1}{x}\right\rfloor+y}\right),
$$

$\mathscr{B}$ is the collection of Borel subsets of $\mathscr{M}$ and $\mu$ is the measure on $\mathscr{M}$ with density function $\frac{1}{\log 2} \frac{1}{(1+x y)^{2}}$ (see [10]). Using ergodicity and the first of the basic relations

$$
\Theta_{n}=\frac{T_{n}}{1+T_{n} V_{n}} \quad \text { and } \quad \Theta_{n-1}=\frac{V_{n}}{1+T_{n} V_{n}},
$$

one gets immediately that

$$
\lim _{n \rightarrow \infty} \frac{1}{n} \#\left\{j \leq n: \Theta_{j}(x)<z\right\}=\mu\left(\mathscr{H}_{z}\right),
$$

where $\mathscr{H}_{z}$ is the subspace of $\mathscr{M}$ consisting of points under the hyperbola

$$
\frac{T}{1+T V}=z \text {. }
$$

The variation we need here is, that instead of using the function $\Theta_{n}$ in every point of the unit square, we consider $B_{n}-1$ functions, namely $\Theta_{n}^{(B)}$ with $0<B<B_{n}$. More precisely, let $B>0$; then the function $\Theta_{n}^{(B)}$ as in $(0.7)$ is defined in $\left(T_{n-1}, V_{n-1}\right) \in[0,1] \times[0,1]$ precisely when the partial quotient $B_{n}$ exceeds $B$, that is, when $T_{n-1} \leq \frac{1}{B+1}$. So $\Theta_{n}^{(B)}$ is defined on the rectangle

$$
\mathscr{R}^{(B)}=\left\{(T, V): 0 \leq T \leq \frac{1}{B+1}, 0 \leq V \leq 1\right\} .
$$

Instead of $(0.6)$ and $(0.7)$ one would like to have formulas expressing $\Theta_{n}^{(B)}$ in terms of $B, T$, and $V$ only. This can be done as follows. Combining (0.4), $(0.6)$, and (0.7), one easily gets

$$
\Theta_{n}^{(B)}=-B^{2} \Theta_{n-1}-B\left(V_{n-1} \Theta_{n-1}-\frac{\Theta_{n-2}}{V_{n-1}}\right)+\Theta_{n-2}
$$

Then use (1.5) to express $\Theta_{n-1}$ and $\Theta_{n-2}$ in terms of $T_{n-1}$ and $V_{n-1}$ and one arrives at

$$
\Theta_{n}^{(B)}=\frac{\left(1-B T_{n-1}\right)\left(B+V_{n-1}\right)}{1+T_{n-1} V_{n-1}} .
$$

This provides the preliminaries for the proof of the following theorem.

(1.9) Theorem. Let $B>0$ be an integer.

(i) For every $x$ and for every $n \geq 1$ such that $0<B<B_{n}$, there holds

$$
\frac{B}{B+1} \leq \Theta_{n}^{(B)}(x) \leq B+1 \text {. }
$$


(ii) For almost all $x$, the sequence $\left\{\Theta_{n}^{(B)}(x)\right\}_{n=1}^{\infty}$ is distributed according to the distribution function

$$
\frac{1}{\log \frac{B+2}{B+1}} G^{(B)}(z)
$$

where

$$
G^{(B)}(z)= \begin{cases}G_{0}^{(B)}(z)=0, & \text { for } z \leq \frac{B}{B+1}, \\ G_{1}^{(B)}(z)=-1+\frac{B+1}{B} z-\log \left(\frac{B+1}{B} z\right), & \text { for } \frac{B}{B+1} \leq z \leq \frac{B+1}{B+2}, \\ G_{2}^{(B)}(z)=\frac{1}{B(B+1)} z+\log \left(\frac{B(B+2)}{(B+1)^{2}}\right), & \text { for } \frac{B+1}{B+2} \leq z \leq B, \\ G_{3}^{(B)}(z)=1-\frac{1}{B+1} z+\log \left(\frac{B+2}{(B+1)^{2}} z\right) & \text { for } B \leq z \leq B+1, \\ G_{4}^{(B)}(z)=\log \frac{B+2}{B+1}, & \text { for } B+1 \leq z .\end{cases}
$$

Proof. From (1.8) we see that $\Theta_{n}^{(B)}<z$ if and only if $\left(T_{n-1}, V_{n-1}\right)$ is in $\mathscr{R}^{(B)}$ and satisfies

or, equivalently,

$$
\frac{\left(1-B T_{n-1}\right)\left(B+V_{n-1}\right)}{1+T_{n-1} V_{n-1}}<z
$$

$$
V_{n-1}<\frac{B^{2} T_{n-1}+z-B}{1-(B+z) T_{n-1}}
$$

So, for given $x$ and fixed $B$ we have to find all pairs $\left(T_{n-1}(x), V_{n-1}(x)\right)$ in $\mathscr{R}^{(B)}$ under the hyperbola

$$
V=\frac{B^{2} T+z-B}{1-(B+z) T} .
$$

Denote by $\mathscr{H}^{(B)}$ the set of points $(T, V)$ under the hyperbola

$$
\mathscr{H}^{(B)}(z): V<\frac{B^{2} T+z-B}{1-(B+z) T} .
$$

Since $\mathscr{R}^{(B)} \cap \mathscr{H}^{(B)}(z)$ is empty for $z<\frac{B}{B+1}$ and $\mathscr{R}^{(B)} \cap \mathscr{H}^{(B)}(z)=\mathscr{R}^{(B)}$ for $z>B+1$, we are done with part (i). For the second part we use the ergodicity given in (1.4), which implies that for almost all $x$ :

$$
\lim _{n \rightarrow \infty} \frac{1}{n} \#\left\{j \leq n: \Theta_{n}^{(B)}(x)<z\right\}=\frac{1}{\mu\left(\mathscr{R}^{(B)}\right)} \mu\left(\mathscr{R}^{(B)} \cap \mathscr{H}^{(B)}(z)\right) .
$$

Therefore, we are left with the computation of $\mu\left(\mathscr{R}^{(B)} \cap \mathscr{H}^{(B)}(z)\right)$ as a function of $z$, which equals, by (1.3),

$$
\frac{1}{\log 2} \iint_{\mathscr{R}^{(B)} \cap \mathscr{H}^{(B)}(z)} \frac{1}{(1+T V)^{2}} d V d T .
$$


For $\frac{B}{B+1} \leq z \leq \frac{B+1}{B+2}$ one gets

$$
\mathscr{R}^{(B)} \cap \mathscr{H}^{(B)}(z)=\left\{(T, V): \frac{B-z}{B^{2}} \leq T \leq \frac{1}{B+1}, 0 \leq V \leq \frac{B^{2} T+z-B}{1-(B+z) T}\right\},
$$

and we find

$$
\begin{aligned}
\frac{1}{\log 2} \iint_{\mathscr{R}^{(B)} \cap \mathscr{P}^{(B)}(z)} \frac{1}{(1+T V)^{2}} d V d T \\
\quad=\frac{1}{\log 2} \int_{\frac{B-z}{B^{2}}}^{\frac{1}{B+1}}\left[\frac{V}{1+T V}\right]_{V=0}^{V=\frac{B^{2} T_{+-B}}{1-(B+z) T}} d T \\
=\frac{1}{\log 2} \int_{\frac{B-z}{B^{2}}}^{\frac{1}{B+1}}\left(\frac{z}{(1-B T)^{2}}-\frac{B}{1-B T}\right) d T \\
=\frac{1}{\log 2}\left[\frac{z}{B(1-B T)}+\log (1-B T)\right]_{\frac{B-z}{B^{2}}}^{\frac{1}{B+1}} \\
=\frac{1}{\log 2}\left(\frac{B+1}{B} z-\log \left(\frac{B+1}{B} z\right)-1\right) .
\end{aligned}
$$

For $\frac{B+1}{B+2} \leq z \leq B$,

$$
\begin{aligned}
\mathscr{R}^{(B)} \cap & \mathscr{H}^{(B)}(z) \\
= & \left\{(T, V): \frac{B-z}{B^{2}} \leq T \leq \frac{B+1-z}{B^{2}+B+z}, 0 \leq V \leq \frac{B^{2} T+z-B}{1-(B+z) T}\right\} \\
& \cup\left\{(T, V): \frac{B+1-z}{B^{2}+B+z} \leq T \leq \frac{1}{B+1}, 0 \leq V \leq 1\right\},
\end{aligned}
$$

and this gives

$$
\begin{aligned}
\frac{1}{\log 2} \iint_{\mathscr{R}^{(B)} \cap \mathscr{P}^{(B)}(z)} \frac{1}{(1+T V)^{2}} d V d T \\
=\frac{1}{\log 2}\left(\frac{z}{B(B+1)}+\log \frac{B(B+1)}{B^{2}+B+z}\right) \\
\quad+\frac{1}{\log 2}\left(\log \frac{B+2}{B+1}-\log \frac{(B+1)^{2}}{B^{2}+B+z}\right) \\
=\frac{1}{\log 2}\left(\frac{z}{B(B+1)}+\log \frac{B(B+2)}{(B+1)^{2}}\right)
\end{aligned}
$$

by a computation similar to the above.

Finally, for $B \leq z \leq B+1$,

$$
\begin{aligned}
\mathscr{R}^{(B)} \cap \mathscr{H}^{(B)}(z)= & \left\{(T, V): 0 \leq T \leq \frac{B+1-z}{B^{2}+B+z}, 0 \leq V \leq \frac{B^{2} T+z-B}{1-(B+z) T}\right\} \\
& \cup\left\{(T, V): \frac{B+1-z}{B^{2}+B+z} \leq T \leq \frac{1}{B+1}, 0 \leq V \leq 1\right\},
\end{aligned}
$$


and the double integral (1.11) equals

$$
\begin{aligned}
\frac{1}{\log 2} & \left(1-\frac{z}{B+1}+\log \frac{(B+1) z}{B^{2}+B+z}\right)+\frac{1}{\log 2}\left(\log \frac{B+2}{B+1}-\log \frac{(B+1)^{2}}{B^{2}+B+z}\right) \\
& =\frac{1}{\log 2}\left(1-\frac{z}{B+1}+\log \frac{B+2}{(B+1)^{2}} z\right) .
\end{aligned}
$$

To find the distribution function $G^{(B)}$, we have to normalize, i.e., we have to divide in each of the cases by

$$
\mu\left(\mathscr{R}^{(B)}\right)=\frac{1}{\log 2} \log \frac{B+2}{B+1} .
$$

This completes the proof of (1.9).

Remark. The special case $B=1$ of Theorem (1.9) yields the result that was found as Lemma 2.24 in [7].

\section{APPROXIMATION BY CONVERGENTS AND MEDIANTS}

In this section we look at the approximation of an irrational number $x$ by all of its mediants and convergents simultaneously.

(2.1) Lemma. Let $G^{(B)}(z)$ be as in (1.9). Then for the function $H(z)$ defined by

$$
H(z)=\sum_{B=1}^{\infty} G^{(B)}(z)
$$

we have

$$
H(z)= \begin{cases}0, & \text { for } z \leq \frac{1}{2}, \\ -1+2 z-\log (2 z), & \text { for } \frac{1}{2} \leq z \leq 1, \\ 1+\log \frac{z}{2}, & \text { for } 1 \leq z .\end{cases}
$$

Proof. Let $G_{i}^{(B)}(z)$ be as in (1.9) for $i=0, \ldots, 4$. Suppose first that $\frac{1}{2} \leq z$ $\leq 1$; let the positive integer $k$ be determined by $\frac{k}{k+1} \leq z<\frac{k+1}{k+2}$. Then

$$
\begin{aligned}
\sum_{B=1}^{\infty} G^{(B)}(z)= & \sum_{B=1}^{k-1} G_{2}^{(B)}(z)+G_{1}^{(k)}(z)+\sum_{B=k+1}^{\infty} G_{0}^{(B)}(z) \\
= & \sum_{B=1}^{k-1}\left(\frac{1}{B(B+1)} z+\log \frac{B(B+2)}{(B+1)^{2}}\right) \\
& +\left(-1+\frac{k+1}{k} z-\log \frac{k+1}{k} z\right)+0 \\
= & \left(1-\frac{1}{k}\right) z+\log \frac{k+1}{2 k}-1+\frac{k+1}{k} z-\log \frac{k+1}{k} z \\
= & -1+2 z-\log 2 z .
\end{aligned}
$$


For $1 \leq z$ we let the integer $k$ be such that $k \leq z<k+1$. Then

$$
\begin{aligned}
\sum_{B=1}^{\infty} G^{(B)}(z)= & \sum_{B=1}^{k-1} G_{4}^{(B)}(z)+G_{3}^{(k)}(z)+\sum_{B=k+1}^{\infty} G_{2}^{(B)}(z) \\
= & \sum_{B=1}^{k-1} \log \frac{B+2}{B+1}+\left(1-\frac{1}{k+1} z+\log \frac{k+2}{(k+1)^{2}} z\right) \\
& +\sum_{B=k+1}^{\infty}\left(\frac{z}{B(B+1)}+\log \frac{B(B+2)}{(B+1)^{2}}\right) \\
= & \log \frac{k+1}{2}+1-\frac{1}{k+1} z+\log \frac{k+2}{(k+1)^{2}} z \\
& +\frac{1}{k+1} z+\log \frac{k+1}{k+2} \\
= & 1+\log \frac{z}{2} .
\end{aligned}
$$

This completes the proof of $(2.1)$.

For any irrational $x$ we introduce the following notation for the collection of all convergents and mediants of $x$ :

$$
\mathscr{A}(x)=\left\{\frac{L}{M}: \frac{L}{M}=\frac{P_{n}}{Q_{n}} \text { or } \frac{L}{M}=\frac{L_{n}^{(B)}}{M_{n}^{(B)}} \text { for some } n, B\right\} .
$$

For any $C>0$ we will denote by $\mathscr{A}^{C}(x)$ the subset

$$
\mathscr{A}^{C}(x)=\left\{\frac{L}{M} \in \mathscr{A}(x): M|M x-L| \leq C\right\}
$$

of $\mathscr{A}(x)$. We enumerate the elements of $\mathscr{A}^{C}(x)$ after ordering them by increasing denominators; thus every fraction $L_{n} / M_{n}$ in $\mathscr{A}^{C}(x)$ is either a convergent or a mediant of $x$, and $M_{i}<M_{j}$ if $i<\hat{j}$.

(2.2) Theorem. Let $C>0$; for almost all $x$

$$
\lim _{n \rightarrow \infty} \frac{1}{n} \#\left\{j \leq n: \frac{L_{j}}{M_{j}} \in \mathscr{A}^{c}(x), M_{j}\left|M_{j} x-L_{j}\right| \leq z\right\}
$$

exists and $\left\{M_{j}\left|M_{j} x-L_{j}\right|: \frac{L_{j}}{M_{j}} \in \mathscr{A}^{C}(x)\right\}$ has limiting distribution $H^{(C)}(z)$ given by

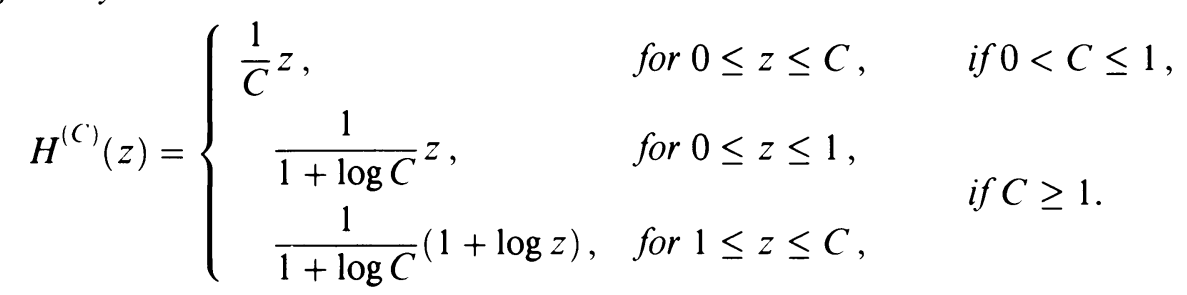

Proof. Let $C>0$ be arbitrary. For $0 \leq z \leq C$ we have to find all $n, B$ (with $\left.0<B<B_{n}\right)$ such that $\Theta_{n}^{(B)}(x) \leq z$ as well as all $n$ for which $\Theta_{n}(x) \leq z$. Let 
$\Lambda^{(B)}(z) \subset \mathscr{R}^{(B)}$ denote the subset for which $\Theta_{n}^{(B)}(x) \leq z$ and let $\Lambda^{(0)}(z)$ be the subset of $[0,1] \times[0,1]$ for which $\Theta_{n}(x) \leq z$. By the ergodicity of $(1.4)$ and the individual ergodic theorem it follows that for almost all $x$

$$
\lim _{n \rightarrow \infty} \frac{1}{n} \#\left\{j \leq n: \Theta_{j}^{(B)}(x) \leq z\right\}=\frac{1}{\mu\left(\mathscr{R}^{(B)}\right)} \mu\left(\Lambda^{(B)}(z)\right)
$$

and

$$
\lim _{n \rightarrow \infty} \frac{1}{n} \#\left\{j \leq n: \Theta_{j}(x) \leq z\right\}=\mu\left(\Lambda^{(0)}(z)\right) .
$$

In (1.9) we saw that

$$
\frac{1}{\mu\left(\mathscr{R}^{(B)}\right)} \mu\left(\Lambda^{(B)}(z)\right)=\frac{1}{\log 2} G^{(B)}(z),
$$

and by $(0.5)$,

$$
\mu\left(\Lambda^{(0)}(z)\right)=\frac{1}{\log 2} F(z) .
$$

Denoting the whole space by $\Lambda_{C}$, these combine to

$$
\begin{aligned}
& \mu\left(\Lambda_{C}\right) \lim _{n \rightarrow \infty} \frac{1}{n} \#\left\{j \leq n: \frac{L_{j}}{M_{j}} \in \mathscr{A}^{C}(x), M_{j}\left|M_{j} x-L_{j}\right| \leq z\right\} \\
& =\frac{1}{\mu\left(\mathscr{R}^{(B)}\right)} \mu\left(\Lambda^{(0)}(z)\right)+\sum_{B=1}^{\infty} \frac{1}{\mu\left(\mathscr{R}^{(B)}\right)} \mu\left(\Lambda^{(B)}(z)\right) \\
& =\frac{1}{\log 2} F(z)+\frac{1}{\log 2} \sum_{B=1}^{\infty} G^{(B)}(z) \\
& =\frac{1}{\log 2} F(z)+\frac{1}{\log 2} H(z)
\end{aligned}
$$

as in (2.1). The distribution function $H^{(C)}(z)$ is now found from the definitions of $F(z)$ and $H(z)$ and by scaling:

$$
H^{(C)}(z)=\frac{F(z)+H(z)}{F(C)+H(C)} .
$$

This proves (2.2).

\section{APPROXIMATION BY NEAREST MEDIANTS}

In this section we look at the approximation of an irrational number $x$ by its nearest mediants, that is, by the mediants with $B=1$ or with $B=B_{n}-1$. Since the case $B=1$ is contained in Theorem (1.9), we look here at $B=B_{n}-1$. Notice that the 'first' mediant $(B=1)$ and the 'final' mediant $\left(B=B_{n}{ }^{-1}\right)$ coincide in case $B_{n}=2$; if $B_{n}=1$, there are no mediants. The first theorem tells us how the final mediants are distributed for a given partial quotient. By

$$
\left\{\left.\Theta_{n}^{\left(B_{n}-1\right)}\right|_{B_{n}=D}\right\}
$$

we will denote the sequence consisting of the $\Theta$ 's belonging to the final mediants for which the partial quotient equals $D$. 
(3.1) Theorem. (i) For every $x$ and for every $n \geq 1$ such that $B_{n} \geq 2$, there holds

$$
\frac{B_{n}-1}{B_{n}} \leq \Theta_{n}^{\left(B_{n}-1\right)} \leq \frac{2 B_{n}}{B_{n}+2} .
$$

(ii) For almost all $x$, the sequence $\left\{\left.\Theta_{n}^{\left(B_{n}-1\right)}\right|_{B_{n}=D}\right\}$ for $D \geq 2$ is distributed according to the distribution function

$$
\frac{1}{\log \frac{(D+1)^{2}}{D(D+2)}} J^{(D)}(z)
$$

where

$$
J^{(D)}(z)=\left\{\begin{array}{l}
J_{0}^{(D)}(z)=0, \quad \text { for } z \leq \frac{D-1}{D}, \\
J_{1}^{(D)}(z)=-1+\frac{D}{D-1} z-\log \left(\frac{D}{D-1} z\right), \\
J_{2}^{(D)}(z)=\frac{1}{D(D-1)} z+\log \left(\frac{(D-1)(D+1)}{D^{2}}\right), \\
\quad \text { for } \frac{D-1}{D} \leq z \leq \frac{D}{D+1}, \\
J_{3}^{(D)}(z)=1-\frac{D+2}{2 D} z+\log \left(\frac{(D+1)^{2}}{2 D^{2}} z\right), \quad \text { for } \frac{2(D-1)}{D+1} \leq z \leq \frac{2(D-1)}{D+1}, \\
J_{4}^{(D)}(z)=\log \frac{(D+1)^{2}}{D(D+2)}, \quad \text { for } \frac{2 D}{D+2} \leq z .
\end{array}\right.
$$

Proof. The proof is an imitation of the proof of Theorem (1.9), the difference being that we have to consider pairs $(T, V)$ here in $\mathscr{R}^{(D-1)} \backslash \mathscr{R}^{(D)}$. We leave the details to the reader.

Let $\mathscr{F}(x)$ denote the collection of final mediants:

$$
\mathscr{F}(x)=\left\{\frac{L}{M}: \frac{L}{M}=\frac{L_{n}^{\left(B_{n}-1\right)}}{M_{n}^{B_{n}-1}} \text { for some } n \text { for which } B_{n} \geq 2\right\} .
$$

We enumerate the elements of $\mathscr{F}(x)$ again after ordering them by increasing denominators; thus every fraction $L_{n} / M_{n}$ in $\mathscr{F}$ is a final mediant of $x$, and $M_{i}<M_{j}$ if $i<j$.

(3.2) Theorem. For almost all $x$

$$
\lim _{n \rightarrow \infty} \frac{1}{n} \#\left\{j \leq n: \frac{L_{j}}{M_{j}} \in \mathscr{F}(x), M_{j}\left|M_{j} x-L_{j}\right| \leq z\right\}
$$


exists and $\left\{M_{j}\left|M_{j} x-L_{j}\right|: \frac{L_{j}}{M_{j}} \in \mathscr{F}(x)\right\}$ has limiting distribution $\frac{1}{\log \frac{3}{2}} J(z)$, where

$$
J(z)= \begin{cases}0, & \text { for } z \leq \frac{1}{2}, \\ -1+2 z-\log (2 z), & \text { for } \frac{1}{2} \leq z \leq \frac{2}{3} \\ \frac{z}{2}+\log \frac{3}{4}, & \text { for } \frac{2}{3} \leq z \leq 1, \\ 1-\frac{z}{2}+\log \left(\frac{3}{4} z\right), & \text { for } 1 \leq z \leq 2, \\ \log \frac{3}{2}, & \text { for } 2 \leq z .\end{cases}
$$

Proof. We have to find all $n$ with $\Theta_{n}^{\left(B_{n}-1\right)}(x) \leq z$. Let $\Lambda^{\left(B_{n}-1\right)} \subset \mathscr{R}^{\left(B_{n}-1\right)}$ denote the subset for which $\Theta_{n}^{\left(B_{n}-1\right)}(x) \leq z$. By the ergodicity of (1.4) and the individual ergodic theorem it follows that for almost all $x$

$$
\lim _{n \rightarrow \infty} \frac{1}{n} \#\left\{j \leq n: \Theta_{j}^{\left(B_{n}-1\right)}(x) \leq z\right\}=\frac{1}{\mu\left(\mathscr{R}^{\left(B_{n}-1\right)} \backslash \mathscr{R}^{\left(B_{n}\right)}\right)} \mu\left(\Lambda^{\left(B_{n}-1\right)}(z)\right) .
$$

From (3.1) we can see that

This gives

$$
\mu\left(\Lambda^{\left(B_{n}-1\right)}(z)\right)=\frac{1}{\log 2} J^{\left(B_{n}-1\right)}(z) .
$$

$$
\begin{aligned}
\lim _{n \rightarrow \infty} & \frac{1}{n} \#\left\{j \leq n: \frac{L_{j}}{M_{j}} \in \mathscr{F}(x), M_{j}\left|M_{j} x-L_{j}\right| \leq z\right\} \\
= & \frac{\sum_{B_{n}-1=1}^{\infty} \mu\left(\Lambda^{\left(B_{n}-1\right)}(z)\right)}{\sum_{B_{n}-1=1}^{\infty} \log \left(\left(B_{n}+1\right)^{2} / B_{n}\left(B_{n}+2\right)\right)}=\frac{1}{\log \frac{3}{2}} \sum_{D=2}^{\infty} J^{(D)}(z) .
\end{aligned}
$$

Suppose first that $\frac{1}{2} \leq z \leq \frac{2}{3}$; then

$$
\begin{aligned}
\sum_{D=2}^{\infty} J^{(D)}(z) & =J_{1}^{(2)}(z)+\sum_{D=3}^{\infty} J_{0}^{(D)}(z) \\
& =-1+2 z-\log (2 z)+0 .
\end{aligned}
$$

Next, let $\frac{2}{3} \leq z \leq 1$; let the positive integer $k$ be determined by $\frac{k-1}{k} \leq z<$ $\frac{k}{k+1}$. Then (just as in the proof of (2.1))

$$
\begin{aligned}
\sum_{D=2}^{\infty} J^{(D)}(z)= & J_{3}^{(2)}(z)+\sum_{D=3}^{k-1} J_{2}^{(D)}(z)+J_{1}^{(k)}(z)+\sum_{D=k+1}^{\infty} J_{0}^{(D)}(z) \\
= & 1-z+\log \left(\frac{9}{8} z\right)+\sum_{D=3}^{k-1}\left(\frac{1}{D(D-1)} z+\log \frac{(D-1)(D+1)}{(D)^{2}}\right) \\
& +\left(-1+\frac{k}{k-1} z-\log \frac{k}{k-1} z\right)+0 \\
= & \frac{z}{2}+\log \frac{3}{4} .
\end{aligned}
$$


For $1 \leq z \leq 2$ we let the integer $k$ be such that $\frac{2(k-2)}{k} \leq z<\frac{2(k-1)}{k+1}$. Then

$$
\begin{aligned}
\sum_{D=2}^{\infty} J^{(D)}(z)= & \sum_{D=2}^{k-2} J_{4}^{(D)}(z)+J_{3}^{(k-1)}(z)+\sum_{D=k}^{\infty} J_{2}^{(D)}(z) \\
= & \sum_{D=2}^{k-2} \log \frac{(D+1)^{2}}{D(D+2)}+\left(1-\frac{k+1}{2(k-1)} z+\log \left(\frac{k^{2}}{2(k-1)^{2}} z\right)\right) \\
& +\sum_{D=k}^{\infty}\left(\frac{z}{D(D-1)}+\log \frac{(D-1)(D+1)}{D^{2}}\right) \\
= & 1+\log \frac{3(k-1)}{2 k}+1-\frac{k+1}{2(k-1)} z+\log \left(\frac{k^{2}}{2(k-1)^{2}} z\right) \\
& +\frac{1}{k-1} z+\log \frac{k-1}{k} \\
= & 1-\frac{z}{2}+\log \frac{3 z}{4} .
\end{aligned}
$$

This completes the proof of (3.2).

Next, we look at the sequence of $\Theta$ 's coming from convergents and nearest mediants of a given $x$. Let $\mathscr{N}(x)$ denote the collection of convergents and nearest mediants:

$$
\mathscr{N}(x)=\left\{\frac{L}{M}: \frac{L}{M}=\frac{P_{n}}{Q_{n}} \text { or } \frac{L}{M}=\frac{L_{n}^{(1)}}{M_{n}^{(1)}} \text { or } \frac{L}{M}=\frac{L_{n}^{\left(B_{n}-1\right)}}{M_{n}^{\left(B_{n}-1\right)}} \text { for some } n\right\},
$$

enumerated in order of increasing denominators $M$.

(3.3) Theorem. For almost all $x$

$$
\lim _{n \rightarrow \infty} \frac{1}{n} \#\left\{j \leq n: \frac{L_{j}}{M_{j}} \in \mathcal{N}(x), M_{j}\left|M_{j} x-L_{j}\right| \leq z\right\}
$$

exists and $\left\{M_{j}\left|M_{j} x-L_{j}\right|: \frac{L_{j}}{M_{j}} \in \mathcal{N}(x)\right\}$ has limiting distribution $\frac{1}{2 \log 2} K(z)$, where

$$
K(z)= \begin{cases}0, & \text { for } z \leq 0 \\ z, & \text { for } 0 \leq z \leq 1 \\ 2-z+2 \log z, & \text { for } 1 \leq z \leq 2 \\ 2 \log 2, & \text { for } 2 \leq z\end{cases}
$$

Proof. We consider convergents and nearest mediants now, so it is clear from their definitions that

$$
K(z)=F(z)+G^{(1)}(z)+J(z)-C(z)
$$

if we denote by $C(z)$ the function that gives the distribution of $\Theta$ 's in case that the first and the final mediants coincide, that is if $B_{n}=2$ (see the remark before Theorem $(3.1))$. To find $C(z)$, we have to evaluate

$$
\mu\left(\left\{\mathscr{R}^{(1)} \backslash \mathscr{R}^{(2)}\right\} \cap \mathscr{H}^{(1)}(z)\right)
$$


(cf. (1.6) and (1.10)). For $z \leq \frac{2}{3}$ this equals

$$
\mu\left(\mathscr{R}^{(1)} \cap \mathscr{H}^{(1)}(z)\right)=G^{(1)}(z)=J(z) .
$$

For $\frac{2}{3} \leq z \leq 1$ we find that

$$
\begin{aligned}
\left\{\mathscr{R}^{(1)} \backslash \mathscr{R}^{(2)}\right\} \cap \mathscr{H}^{(1)}(z)= & \left\{(T, V): \frac{1}{3} \leq T \leq \frac{2-z}{2+z}, 0 \leq V \leq \frac{T+z-1}{1-(1+z) T}\right\} \\
& \cup\left\{(T, V): \frac{2-z}{2+z} \leq T \leq \frac{1}{2}, 0 \leq V \leq 1\right\},
\end{aligned}
$$

and a straightforward calculation of

$$
\frac{1}{\log 2} \iint_{\mathscr{R}^{(B)} \cap \mathscr{H}^{(B)}(z)} \frac{1}{(1+T V)^{2}} d V d T
$$

in this case, as in the proof of (1.9), leads to

$$
C(z)= \begin{cases}0, & \text { for } z \leq \frac{1}{2} \\ -1+2 z-\log (2 z), & \text { for } \frac{1}{2} \leq z \leq \frac{2}{3} \\ 1-z+\log \left(\frac{9}{8} z\right), & \text { for } \frac{2}{3} \leq z \leq 1 \\ \log \frac{9}{8}, & \text { for } 1 \leq z\end{cases}
$$

If we use this with (0.5), Theorems (1.9) and (3.2) in (3.4) we immediately get the function $K(z)$ as in the statement of the theorem.

(3.5) Remarks. In [4], Ito proved the part of (3.3) with $z \leq 1$. Using this, he was able to prove that for $0 \leq \lambda \leq 1$ :

$$
\lim _{n \rightarrow \infty} \frac{1}{\log n} \#\left\{(p, q)|| x-\frac{p}{q} \mid<\frac{\lambda}{q^{2}} \text { with } \operatorname{gcd}(p, q)=1 \text { and } q \leq n\right\}=\frac{12}{\pi^{2}} \lambda
$$

(for almost all $x$ ). In fact, this holds for arbitrary $\lambda \geq 0$ and is known as Erdös' theorem (see [2]). Jager proved all of Theorem (3.3) in [7]; there, he also gives an alternative proof for the part of Erdös' theorem with $0 \leq \lambda \leq 1$, using Fatou's theorem (see $\S 4$ below). Notice that $K(z)=2 F\left(\frac{z}{2}\right)$.

\section{TheOREMS OF LEGENDRE AND FATOU}

The linear part in the distribution function $F$ of $(0.5)$ for $0 \leq z \leq \frac{1}{2}$ reflects the fact that the convergents to any $x$ include all rationals $P / Q$ for which $Q|Q x-P|<\frac{1}{2}$; this is known as Legendre's theorem, and it is part (i) of Theorem (4.1) below, cf. [5, 2, 4]. Since the distribution function in (3.3) is linear up to $z=1$, one wonders whether this indicates that for every $x$ all rationals satisfying $Q|Q x-P|<1$ are among the set of convergents and nearest mediants to $x$. This is indeed the case, and it seems that this was first observed in [3], where it is stated without proof. The first proof, apparently, appeared in a paper by Koksma (see [8 and 9]). Fatou's theorem is part (ii) of Theorem (4.1) below. 
(4.1) Theorem. Let $x$ be an irrational number and $P / Q$ a rational number $(Q>0$ and $\operatorname{gcd}(P, Q)=1)$.

(i) If $Q|Q x-P|<\frac{1}{2}$, then

$$
\frac{P}{Q}=\frac{P_{n}(x)}{Q_{n}(x)} \text { for some } n \geq 0 \text {. }
$$

(ii) If $Q|Q x-P|<1$, then

$$
\frac{P}{Q}=\frac{B P_{n-1}(x)+P_{n-2}(x)}{B Q_{n-1}(x)+P_{n-2}(x)} \text { for some } n \geq 2 \text { and } B \in\left\{0,1, B_{n}-1\right\} \text {. }
$$

Proof. The proof consists of two parts; first we show (using Koksma's argument) that if $\frac{P}{Q}$ is not a convergent or mediant, then necessarily $Q|Q x-P|>1$. For, in this case we can find integers $n>0$ and $B\left(0 \leq B<B_{n}\right)$ such that $\frac{P}{Q}$ lies between

$$
\frac{P^{\prime}}{Q^{\prime}}=\frac{B P_{n-1}+P_{n-2}}{B Q_{n-1}+Q_{n-2}} \text { and } \frac{P^{\prime \prime}}{Q^{\prime \prime}}=\frac{(B+1) P_{n-1}+P_{n-2}}{(B+1) Q_{n-1}+Q_{n-2}} .
$$

If we assume (the other case being similar) that $\frac{P}{Q}<x$, then

$$
\frac{P^{\prime}}{Q^{\prime}}<\frac{P}{Q}<\frac{P^{\prime \prime}}{Q^{\prime \prime}}<x
$$

This implies

$$
\frac{1}{Q Q^{\prime}} \leq \frac{P}{Q}-\frac{P^{\prime}}{Q^{\prime}}<\frac{P^{\prime \prime}}{Q^{\prime \prime}}-\frac{P^{\prime}}{Q^{\prime}}=\frac{P_{n-1} Q_{n-2}-P_{n-2} Q_{n-1}}{Q^{\prime} Q^{\prime \prime}}=\frac{1}{Q^{\prime} Q^{\prime \prime}}
$$

since $P_{n-1} Q_{n-2}-P_{n-2} Q_{n-1}=1$. So we see that $Q>Q^{\prime \prime}$.

But on the other hand,

$$
\frac{1}{Q Q^{\prime \prime}} \leq \frac{P^{\prime \prime}}{Q^{\prime \prime}}-\frac{P}{Q}<x-\frac{P}{Q},
$$

so if

$$
x-\frac{P}{Q}<\frac{1}{Q^{2}},
$$

we would get

$$
\frac{1}{Q Q^{\prime \prime}}<\frac{1}{Q^{2}}
$$

and thus $Q^{\prime \prime}>Q$, a contradiction.

In the second part of the proof we therefore consider only convergents and mediants of $x$. By (1.9)(i) we have $\Theta_{n}^{(B)}>\frac{1}{2}$ for any $n$ if $B>0$; this finishes the proof of (4.1)(i).

It remains to prove that $Q|Q x-P|<1$ can only hold for convergents and nearest mediants; thus suppose that

$$
Q|Q x-P|<1
$$


and suppose, moreover, that $B \geq 2$ in

$$
\frac{P}{Q}=\frac{B P_{n-1}+P_{n-2}}{B Q_{n-1}+Q_{n-2}} \text {. }
$$

We will show that in that case, $B=B_{n}-1$.

By (1.8), the inequality $Q|Q x-P|=\Theta_{n}^{(B)}<1$ is equivalent to

$$
\left(1-B T_{n-1}\right)\left(B+V_{n-1}\right)<1+T_{n-1} V_{n-1} \text {. }
$$

Then

$$
T_{n-1}>\frac{B+V_{n-1}-1}{B^{2}+B V_{n-1}+V_{n-1}}>\frac{B-1}{B^{2}}
$$

since $\frac{B+V-1}{B^{2}+B V+V}$ increases monotonically with $V(V>0)$. This implies

$$
\frac{1}{B_{n}+T_{n}}=T_{n-1}>\frac{B-1}{B^{2}}=\frac{1}{B+1+\frac{1}{B-1}},
$$

so

$$
B_{n}<B_{n}+T_{n}<B+1+\frac{1}{B-1} \leq B+2,
$$

in which the last inequality follows from our assumption that $B \geq 2$. Thus we see that $B>B_{n}-2$, and since by definition $B<B_{n}$, we find that $B=B_{n}-1$. This completes the proof of (4.1).

\section{ACKNOWLEDGMENT}

This research was done while the author was supported by the Nederlandse organisatie voor wetenschappelijk onderzoek NWO.

\section{BIBLIOGRAPHY}

1. W. Bosma, H. Jager, and F. Wiedijk, Some metrical observations on the approximation by continued fractions, Indag. Math. 45 (1983), 281-299.

2. P. Erdös, Some results on Diophantine approximation, Acta Arith. 5 (1959), 359-369.

3. P. Fatou, Sur l'approximation des incommensurables et les séries trigonométriques, C. R. Acad. Sci. Paris 139 (1904), 1019-1021.

4. S. Ito, Algorithms with mediant convergents and their metrical theory, Preprint.

5. S. Ito and H. Nakada, On natural extensions of transformations related to Diophantine approximations, Proceedings of the Conference on Number Theory and Combinatorics, World Scientific, Singapore, 1985.

6. H. Jager, The distribution of certain sequences connected with the continued fraction, Nederl. Akad. Wetensch. Proc. Ser. A 89 (1986), 61-69.

7. __ Some metrical observations on the approximation of an irrational number by its nearest mediants, Preprint.

8. J. F. Koksma, Bewijs van een stelling over kettingbreuken, Mathematica A 6 (1937/38), 226231 .

9. __ On continued fractions, Simon Stevin 29 (1951/52), 96-102.

10. H. Nakada, S. Ito, and S. Tanaka, On the invariant measure for the transformations associated with some real continued fractions, Keio Engrg. Rep. 30 (1977), 159-175.

Department of Pure Mathematics, University of Sydney, Sydney NSW 2006, AusTralia. E-mail: wieb@galois.math.su.oz.au 